DOI: 10.12731/2658-6649-2020-12-5-97-112

УДК 612.42

\title{
ВЛИЯНИЕ ЭКЗАМЕНАЦИОННОГО СТРЕССА НА АМПЛИТУДУ ВОЛН ЭЭГ В ТЕМЕННОЙ ДОЛЕ ГОЛОВНОГО МОЗГА У СТУДЕНТОВ ФАКУЛЬТЕТА БИОЛОГИИ-ХИМИИ С РАЗЛИЧНЫМИ ИНДИВИДУАЛЬНО-ТИПОЛОГИЧЕСКИМИ ОСОБЕННОСТЯМИ
}

\section{Рустамова T.B.}

Во время эмоционального напряжения основным показателем функиионального состояния ЦНС является изменение амплитудно-частотной характеристики ЭЭГ. В связи с этим, выявление некоторых аспектов нейрофизиологических механизмов формирования этих состояний во время экзаменационного процессапредставляет значительный интерес как с теоретической, так и с практической точки зрения. Кроме этого, важно изучение возрастныхособенностейданных изменений и интеграционной реактивности ЭЭГ-коррелятв периоды эмоционального напряжения $и$ изменения физиологических функиий.

Цель. Сравнительное изучение возрастных закономерностей динамики амплитудь альфа-, бета-, дельта- и тета-волн ЭЭГ, характеризующих электрическую активность теменной доли головного мозга, во время эмоционального напряжения в условиях экзаменационного стресса у студентов I-го и V-го курса факультета биологии-химии с различными типами темперамента.

Материалы и методы. Исследования проводились за 2 месяча до экзаменов в обычные дни (ОД), за 30 мин до экзамена (ДЭ) и через 30 мин после экзамена (ПЭ).Перед началом исследования у студентов определялся тип темперамента нервной системы по тестам Г.Айзенка.Ситуативная тревожность и индивидуальное тревожное состояние определялось в трех разных ситуацияхпо Д.Д. Спилбергу с помощью экспресс версии тест-анкет. Электрофизиологические исследования включали проведение ЭЭГкоры теменной доли мозга (Р правой и левой) в течении 10-12 минут. Результатыл.Установлено, что в зависимости от темперамента нервной системы у студентов I-го и V-го курса имеется статистически достоверная разница 
в показателях электрической активности мозга. Кроме того, существует статистически достоверная разница между группами.У студентов с меланхолическим типом в том же возрасте при сравнении периодов ОД, ДЭ и ПЭ выявлены статистически достоверные различия в показателях ЭЭГ. У 17-летних студентов меланхолического типа в периоды ДЭ и ПЭ, в каждой из парных (левой и правой)теменных долей головного мозга от воздействия эмочуиональной напряженности тревожной ситуацุии амплитуда ЭЭГ дельта-, тета- и альфа-волн вІ семестре изменяется по нарастающуей. Однако у тех же студентов бета-волны изменяются двухфазно. Во время экзаменационного прочесса от воздействия эмоционального напряжения у студентов меланхолического типа в левой и правой теменных долях головного мозга в ритме амплитуды волн ЭЭГ резких отклонений не происходит. В экзаменационной ситуации у студентов $V$-го курсав отличие от студентов I-го курса нейрофизиологический коррелят эмоционального напряжения в зависимости от изменений активности в разных участках долей головного мозга между разными группами не различается.

Заключение. В зависимости от силь, подвижности и стабильности нервной системы изменение ритмов амплитуды волн ЭЭГголовного мозга в период ДЭ и после имеет различный характер. В амплитуде больиинства волн в период ПЭ наблюдается частичное снижение.

Ключевые слова: эмочия; ситуативная тревожность; теменная доля головного мозга; иентральная нервная система.

\section{INFLUENCE OF EXAMINATION STRESS ON EEG AMPLITUDE IN THE CEREBRAL SPHERE OF THE BRAIN OF STUDENTS OF THE FACULTY OF BIOLOGY-CHEMISTRY WITH DIFFERENT INDIVIDUAL TYPOLOGICAL FEATURES}

\section{Rustamova T.V.}

During emotional stress, the main indicator of the functional state of the central nervous system is the change in the amplitude-frequency characteristics of the EEG. In this regard, some aspects of the neurophysiological mechanisms of the formation of this situation during the exam, from the point of view of both theoretical and applied nature, are of great interest. In addition, the age-related aspects of these changes and EEG correlates during the emotional situation of 
various physiological functions and the study of their integration reactivity is of great importance.

Purpose. Research comparative study of age-related patterns of changes in dnamics during the emotional stress of the examination process EEG-amplitude of electrical activity of alpha, beta, delta and theta waves in the parietal lobe of the brain in 1st and 5th year students of the Faculty of Biology and Chemistry with different types of temperament.

Materials and methods. The studies were carried out 2 months before exams on ordinary days (ND), 30 minutes before the exam (BE) and 30 minutes after the exam $(A E)$. Before the start of the study, the type of temperament of the nervous system was determined in students according to the tests of $G$. Aysenck. Situational anxiety and individual anxiety was determined in three different situations according to D.D. Spielberg using the express version of the test questionnaires. In electrophysiological studies, an EEG of the cerebral cortex of the parietal lobe (P right and left) was carried out for 10-12 minutes.

Results: It was found that, depending on the temperament of the nervous system in 1st and 5th year students, there is a significant difference between different types in the electrical activity of the brain. In addition, there is a reliable difference between groups in terms of statistics. In contrast, at the same age, when comparing the periods of $N D, B E$ and $A E$ in the melancholic type, statistical reliability was obtained. In 17-year-old students of the melancholic type in the period of $B E$ and $A E$, in each of the paired (left and right) parietal lobes of the brain, the amplitude of the EEG delta, theta and alpha waves of the first semester changes along an increasing line due to the influence of emotional tension in an anxious situation. However, for the same students, beta waves change in two phases. During the examination process, there are no sharp deviations in the rhythm of the amplitude of the EEG waves in the left and right parietal lobes of the brain in the left and right parietal lobes of the melancholic type from the influence of emotional stress. In the examination situation in 5th year students, the neurophysiological correlator of emotional stress depending on changes in activity in different in the lobes of the brain, in contrast to 1 st year students, there are no changes in the same indicators between different groups.

Conclusion: Depending on the strength, mobility and stability of the nervous system, changes in the rhythms of the amplitude of the EEG brain waves during the DE period and after have a different character. In the amplitude of most PE waves, partial decreases are observed.

Keywords: emotion; situational anxiety; parietal lobes of the brain; central nervous system. 


\section{Введение}

В процессе учебной деятельности студентов экзаменационная ситуация является важным социальным фактором, формирует мотивационную деятельность и создает выраженную эмоциональную напряженность [3, 16]. В современном мире на разных курсах высших учебных заведений интенсификация учебной программы и резкое изменение содержания и формы экзаменационного процесса вызывают изменения в высшей нервной деятельности, связанные с новыми требованиями, что обуславливает также изменения ряда физиологических функций и их интеграции и приводит к возникновению эмоционального стресса.

Ряд исследователей увеличение частоты нервных расстройств у подростков и молодежи связывают в первую очередь с воздействием негативных психосоциальных факторов и нервным напряжением от интенсификации учебной программы $[2,7,14]$. С этой точки зрения экзаменационный стресс является основным фактором, создающим психофизиологическое напряжение $[8,10,11,12,16,19]$.

Экзаменационный стресс, сопровождаемый появлением комплекса психофизиологических изменений, оказывает резкое воздействие на жизнедеятельность, образование, трудовую и спортивную деятельность и т.д. Так, развивающийся у студентов перед экзаменом «синдром ожидания», способствующий возникновению психофизиологического стресса, наряду с тем, что является причиной увеличения тревожной ситуации $[11,16,19]$, способствует также нарушениям адаптации к стрессовым условиям в результате ослабления регуляции вегетативно-гормонального функционирования и работы других систем организма $[2,5,12,16,18]$. Во время эмоционального напряжения основным показателем функционального состояния ЦНС является изменение амплитудно-частотной характеристики ЭЭГ $[13,14,15,17]$. В связи с этим, выявление некоторых аспектов нейрофизиологических механизмов формирования этих состояний во время экзаменационного процесса представляет значительный интерес как с теоретической, так и с практической точки зрения [2, $4,6,8,10,11]$. Кроме этого, важно изучение возрастных особенностей данных изменений и интеграционной реактивности ЭЭГ-коррелят в периоды эмоционального напряжения и изменения физиологических функций $[2,7,9,15]$.

Целью настоящего исследования явилось сравнительное изучение возрастных закономерностей динамики амплитуды альфа-, бета-, дельта- и тета- волн ЭЭГ, характеризующих электрическую активность теменной доли головного мозга, во время эмоционального напряжения в условиях экзаменационного стресса у студентов I-го и V-го курса факультета биологии-химии с различными типами темперамента. 


\section{Материалы и методы}

Исследование проведено на факультете биологии-химииГянджинского Государственного Университета. К исследованию на добровольной основе были привлечены обучающиеся по методике курикулумана получение степени бакалаврапрактически здоровые студенты 17-ти лет и 21-го года (n=68). 32 студента были в возрасте 17 лет, 36 студентов - в возрасте 21 года. Исследования проводились в трех группах на протяженииучебного процесса (за 2 месяца до экзаменов), за 30 минут до экзаменов и спустя 30 минут после экзаменов. Перед началом исследования у студентов определялся тип темперамента нервной системы по тестам Г. Айзенка[1]. Ситуативная тревожность и индивидуальное тревожное состояние определялось в трех разных ситуациях: обычный день (ОД), перед экзаменом (ДЭ) и после экзамена (ПЭ) по Д.Д Спилбергу [20] с помощью экспресс версии тест-анкет. Среди нейрофизиологических методов определения степени тревожности головного мозга, одно из важных мест занимает запись электрической активности мозга -электроэнцефалография (ЭЭГ). Электрофизиологические исследования осуществлялись в 6 монополярныхотведениях с активными электродами, расположенными по международной системе «10-20»: регистрировалась ЭЭГ коры теменной доли мозга (Р правой и левой) в течении 10-12 минут в трех ситуациях (ОД, ДЭ и ПЭ), референтный электрод размещался на ипсилатеральной части мочки уха. Регистрация проводилась с помощью аппарат-комплекса набора электродов «Нейрон-спектр 16А» («Neyrosoft» MMC, İvanovo), полученные ЭЭГ-волны посредством метода Фурье (скоростной обмен) подвергались амплитудно-спектральному анализу. В каждом отведении для каждого диапазона амплитуды ритма (дельта, тета, альфа, бета) вычислялись процентный показатель и показатели спектральной силы, сила спектра для диапазона амплитуд (согласно показаниям Нейрософта). Время анализа - 8 секунд. С учетом количества обследованных статистическая обработка проводилась непараметрическими методами с помощью программыSPSS (Statistical Package for Social Science). В то же время, учитывая необходимость измерения состояния тревожности в трех различных состояниях и сравнение в трех формах (сравнение между двумя группами, между несколькими группами, внутри группы), для парных выборок использовался критерий Wilcoxon,между двумя независимыми выборками - критерий Mann-Whitney и для сравнения между двумя разными выборками использовался критерий ANOVA - F. Все цифровые данные, полученные в ходе исследования, были подвергнуты статистической обработке с учетом современных рекомендаций. Расчеты проведены в электронной таблице EXCEL. 


\section{Результаты}

Было установлено, что среди 17-летних первокурсников распределение по типам темперамента нервной системы было следующим: 4 флегматика, 10 холериков, 10 сангвиников и 8 меланхоликов. Среди21-летних V-курсников было 5 флегматиков, 13 холериков, 11 сангвиников и 7 меланхоликов. В первом варианте проведенного исследования у 17-летних студентов I курса изучены происходящие под действием эмоционального напряжения экзаменационного процессаизменения в амплитуде волн ЭЭГ теменных долей головного мозга. Данные результаты представлены в таблице 1.

Таблицча 1.

Сравнительный анализ амплитудных показателей волн ЭЭГ левого и правого участка теменной доли головного мозга по различным параметрам у 17-летних студентов меланхолического типа по сравнению с другими типами

\begin{tabular}{|c|c|c|c|c|c|c|c|c|c|c|c|c|c|}
\hline \multirow{11}{*}{$\begin{array}{l}\mathrm{P} \\
\text { левая }\end{array}$} & & \multicolumn{4}{|c|}{ Обычный день } & \multicolumn{4}{|c|}{ До экзамена } & \multicolumn{4}{|c|}{ После экзамена } \\
\hline & & Дельта & Тета & Альфа & Бета & Дельта & Тета & Альфа & Бета & Дельта & Тета & Альфа & Бета \\
\hline & $\mathrm{M}$ & 184,0 & 134,5 & 74,3 & 60,1 & 234,9 & 159,8 & 82,5 & 21,0 & 242,3 & 172,4 & 122,5 & 55,5 \\
\hline & $\pm \mathrm{m}$ & 32,0 & 15,2 & 4,3 & 9,0 & 24,7 & 9,2 & 15,8 & 3,5 & 17,4 & 4,1 & 21,2 & 12,5 \\
\hline & $\min$ & 75 & 63 & 58 & 22 & 70 & 100 & 52 & 11 & 150 & 156 & 61 & 20 \\
\hline & $\max$ & 265 & 170 & 90 & 90 & 278 & 190 & 190 & 40 & 285 & 190 & 195 & 99 \\
\hline & род & & & & & 0,141 & 0,091 & 0,310 & 0,017 & 0,051 & 0,025 & 0,128 & 0,944 \\
\hline & рдэ & & & & & & & & & 0,889 & 0,183 & 0,161 & 0,036 \\
\hline & $\mathrm{pf}$ & 0,670 & 0,932 & 0,061 & 0,104 & 0,171 & 0,234 & 0,017 & 0,308 & 0,392 & 0,012 & 1,000 & 0,932 \\
\hline & $\mathrm{px}$ & 0,119 & 0,010 & 0,395 & 0,328 & 0,964 & 0,721 & 0,065 & 0,082 & 0,858 & 0,789 & 0,859 & 0,475 \\
\hline & ps & 0,789 & 0,150 & 0,929 & 0,054 & 0,100 & 0,054 & 0,109 & 0,032 & 0,081 & 0,003 & 0,561 & 0,266 \\
\hline \multirow{9}{*}{$\begin{array}{l}\text { P } \\
\text { пра- } \\
\text { вая }\end{array}$} & $M$ & 183,8 & 133,4 & 71,1 & 32,9 & 226,0 & 158,0 & 71,8 & 21,1 & 237,6 & 170,4 & 77,0 & 27,3 \\
\hline & $\pm \mathrm{m}$ & 33,3 & 17,6 & 2,7 & 8,9 & 24,2 & 10,3 & 5,3 & 3,3 & 20,2 & 5,7 & 6,1 & 6,2 \\
\hline & $\min$ & 70 & 50 & 64 & 12 & 65 & 95 & 56 & 12 & 130 & 145 & 52 & 14 \\
\hline & $\max$ & 269 & 179 & 80 & 80 & 265 & 198 & 100 & 39 & 289 & 194 & 95 & 69 \\
\hline & род & & & & & 0,237 & 0,128 & 0,624 & 0,203 & 0,051 & 0,050 & 0,441 & 0,889 \\
\hline & рдэ & & & & & & & & & 0,889 & 0,183 & 0,161 & 0,036 \\
\hline & $\mathrm{pf}$ & 0,495 & 0,932 & 0,228 & 0,305 & 0,341 & 1,000 & 0,033 & 0,497 & 0,147 & 0,017 & 0,050 & 0,060 \\
\hline & $\mathrm{px}$ & 0,197 & 0,036 & 0,211 & 0,306 & 0,195 & 0,657 & 0,037 & 0,075 & 0,824 & 0,722 & 0,964 & 0,532 \\
\hline & ps & 1,000 & 0,534 & 0,928 & 0,533 & 0,153 & 0,049 & 0,247 & 0,109 & 0,351 & 0,037 & 0,398 & 0,130 \\
\hline
\end{tabular}

Примечание: Статистическая достоверность между показателями:

род - в той же группе с показателями обычного дня (парное - по критерию Wilcoxon); рдэ - в той же группе с показателями перед экзаменами (парное - по критерию Wilcoxon). pf - с показателями группы флегматиков (ранговое, по критерию Mann-Whitney); px - с показателями группы холериков (ранговое, по критерию Mann-Whitney); ps - с показателями группы сангвиников (ранговое, по критерию Mann-Whitney); 
Как видно из таблицы, у 17-летних студентовв сравнении с ОДв период ДЭ в левой части теменной доли головного мозга амплитуда волн ЭЭГ была выше на 27,7\%, а в период ПЭ амплитуда волн ЭЭГ увеличилась еще больше - на $31,7 \%$ ( $<0,01)$. Однако, это увеличение в амплитуде по сравнению с ДЭ составило всего 4\%. Аналогичные закономерности наблюдались в амплитуде волн ЭЭГ и в правой части теменной доли головного мозга. Так, если в сравнении с ОД амплитуда дельта-волн ДЭ увеличилась на $23,0 \%$, то в период ПЭ этот показатель увеличился еще значительней $-29,3 \%(\mathrm{P}<0,01)$.Тем не менее, увеличение в амплитуде по сравнению с ДЭ составило всего 6,3\%. Как можно заметить, у 17-летних студентов в обеих частях теменной доли головного мозга под воздействием тревожной ситуации эмоционального напряжения в период ДЭ и ПЭ амплитуда дельта-волн ЭЭГ резко увеличивается, однако это увеличение более существенно в период ПЭ (таб.1). Эти результаты показывают, что во время экзаменов в каждой из двух частей теменной доли головного мозга изменение амплитуды дельта-волн ЭЭГ у 17-летних студентов в течение I семестра происходит по нарастающей линии. В режиме ожидания результатов ПЭ на амплитуду дельта-волн тревожная ситуация воздействует еще резче.

У молодых людей того же возраста повышение амплитуды тета-волн ЭЭГ левого участка теменной долипри ДЭ в сравнении с ОДсоставило $18,8 \%$. Это различие в период ПЭ было еще более заметным и по сравнению с ОД было выше на 28,2\% (таб. 1). В течении экзаменационного процесса разница составила $9,4 \%$. В соответствии со всем этим у студентов наблюдается повышение амплитуды тета-волн ЭЭГ в правой части теменной доли головного мозга. Так, во время каждого из двух периодов экзаменационного процесса амплитудатета-волн ЭЭГ по сравнению с ОД повышалась на $18,4-27,7 \%$, соответственно $(\mathrm{P}<0,01)$ (таб.1).

Особо надо отметить, что несмотря на значительно более низкие абсолютные значения амплитуды тета-волн ЭЭГ в сравнении с абсолютным значением дельта-волн, относительные изменения имеют сходный характер (таб.1).

У 17-летних студентов амплитуда альфа-волн ЭЭГ левой теменной

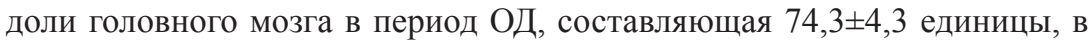
период ДЭ, увеличившись на $10 \%$, стала равной $82,5 \pm 15,8$ единицы. Этот уровень в период ПЭ по сравнению с ОД, увеличился на 64,8 \% исоставил $122,5 \pm 21,2$ единиц (таб.1). 
Амплитуда альфа-волн ЭЭГ правого участка теменнойдоли головного мозга в период ОД была 71,1 2,7 единиц, в период ДЭ эта величина рав-

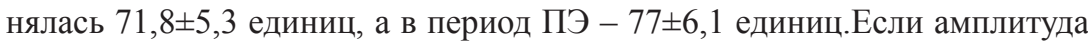
альфа-волн ЭЭГправой теменной доли головного мозга в период ДЭ не изменялась, то в период ПЭ отмечались незначительные отклонения (увеличение на $8,3 \%$ ).

У студентов этого возраста амплитуда бета-волн ЭЭГ левого участка теменной доли головного мозга по сравнению с ОД в период ДЭ резко снизилась (примерно в 3 раза, $\mathrm{P}<0,01$ ).

Однако несмотря на то, что это снижениев период ПЭ приближалось к уровню ОД, оно все еще было выше уровня ДЭ более, чем в 2 раза (таб.1).Абсолютный показатель амплитуды бета-волн ЭЭГ правого участка теменной доли головного мозга по сравнению с левой долейбыла ниже и равнялась $32,9 \pm 8,9$ в период ОД, $21,1 \pm 3,3$ в период ДЭ и $27,3 \pm 6,2$ в период ПЭ.

Эти результаты показывают, что у студентов I-го курса в период экзаменационного процесса изменения амплитуды бета-волн ЭЭГ в каждом из двух участков теменной доли головного мозга носят волнообразный характер. Усиление волнения в период ДЭ и напряженность, связанная с ожиданием результата экзамена, оказывая резкое воздействие на амплитуду данных волн, приводит к их снижению. Однако это снижение в период ПЭ приближается к уровню ОД.

В очередном варианте проводимого исследования, изменения в амплитуде волн ЭЭГ теменной доли головного мозга, происходящие под воздействием эмоциональной напряженности экзаменационного процесса, изучались у 21-летних студентов V курса. Данные результаты представлены в таблице 2.

Установлено, что у 21-летних студентов амплитуда волн ЭЭГ левой части теменной доли в каждой из трех групп практически не изменяется. Это показывает, что эмоциональный стресс, возникающий от экзаменационного волнения устудентов-меланхоликов V-го курса, не оказывая серьезного воздействия на электрическую активность волн ЭЭГ, не демонстрирует различий при переходе от одной волны к другой.

Аналогичные закономерности обнаруживаются и у молодых людей в возрасте 21 года в амплитуде волн ЭЭГ правой части теменной доли головного мозга. Так, здесь также в каждой из трех групп амплитуда волн ЭЭГ практически не изменяется. 
Таблицуа 2.

Сравнительный анализ амплитудных показателей ЭЭГ левого и правого участка теменной доли головного мозга по различным параметрам 21-летних студентов меланхолического типа по сравнению с другими типами

\begin{tabular}{|c|c|c|c|c|c|c|c|c|c|c|c|c|c|}
\hline \multirow{12}{*}{$\begin{array}{l}\text { Р } \\
\text { левая }\end{array}$} & & \multicolumn{4}{|c|}{ Обычный день } & \multicolumn{4}{|c|}{ До экзамена } & \multicolumn{4}{|c|}{ После экзамена } \\
\hline & & Дельта & Тета & Альфа & Бета & Дельта & Тета & Альфа & Бета & Дельта & Тета & Альфа & Бета \\
\hline & M & 259,4 & 165,1 & 76,4 & 18,0 & 266,0 & 169,0 & 76,4 & 16,7 & 262,3 & 166,3 & 76,3 & 18,0 \\
\hline & $\pm \mathrm{m}$ & 3,1 & 4,6 & 5,5 & 2,6 & 3,7 & 6,6 & 4,1 & 2,0 & 2,8 & 3,0 & 3,1 & 2,5 \\
\hline & $\min$ & 250 & 155 & 65 & 12 & 258 & 150 & 65 & 10 & 249 & 155 & 65 & 10 \\
\hline & $\max$ & 270 & 190 & 100 & 30 & 282 & 196 & 98 & 25 & 270 & 177 & 89 & 28 \\
\hline & p17 & 0,182 & 0,352 & 0,826 & 0,004 & 0,684 & 0,954 & 0,562 & 0,487 & 1,000 & 0,246 & 0,147 & 0,011 \\
\hline & род & & & & & 0,176 & 0,553 & 0,833 & 0,866 & 0,601 & 0,753 & 0,753 & 1,000 \\
\hline & рдэ & & & & & & & & & 0,612 & 0,866 & 1,000 & 1,000 \\
\hline & $\mathrm{pf}$ & 0,463 & 0,222 & 0,682 & 0,414 & 0,462 & 0,808 & 0,684 & 0,569 & 0,625 & 0,255 & 0,568 & 0,511 \\
\hline & $\mathrm{px}$ & 0,779 & 0,633 & 0,634 & 0,361 & 0,661 & 0,721 & 0,251 & 0,812 & 0,552 & 0,499 & 0,283 & 1,000 \\
\hline & ps & 0,617 & 0,238 & 0,466 & 0,338 & 0,525 & 0,319 & 0,219 & 0,276 & 0,651 & 0,341 & 0,358 & 0,133 \\
\hline \multirow{10}{*}{$\begin{array}{l}\text { P } \\
\text { пра- } \\
\text { вая }\end{array}$} & M & 264,6 & 164,7 & 78,3 & 18,1 & 268,9 & 169,7 & 77,6 & 18,0 & 265,6 & 167,6 & 76,0 & 18,7 \\
\hline & $\pm \mathrm{m}$ & 3,5 & 4,6 & 5,2 & 2,1 & 4,1 & 5,9 & 3,8 & 2,8 & 2,7 & 2,8 & 3,0 & 2,7 \\
\hline & $\min$ & 254 & 150 & 63 & 11 & 256 & 158 & 63 & 10 & 255 & 156 & 63 & 11 \\
\hline & $\max$ & 278 & 185 & 99 & 28 & 286 & 200 & 91 & 28 & 275 & 180 & 85 & 29 \\
\hline & p17 & 0,183 & 0,487 & 0,413 & 0,385 & 0,027 & 0,562 & 0,295 & 0,416 & 0,817 & 0,602 & 0,728 & 0,202 \\
\hline & род & & & & & 0,398 & 0,498 & 0,933 & 0,735 & 0,799 & 0,735 & 0,553 & 0,752 \\
\hline & рдэ & & & & & & & & & 0,611 & 0,671 & 0,686 & 1,000 \\
\hline & $\mathrm{pf}$ & 0,569 & 0,463 & 0,121 & 0,464 & 0,743 & 0,624 & 0,807 & 0,935 & 0,463 & 0,413 & 0,513 & 0,413 \\
\hline & $\mathrm{px}$ & 0,809 & 0,721 & 0,781 & 0,449 & 0,905 & 0,842 & 0,111 & 0,721 & 0,634 & 0,841 & 0,163 & 0,721 \\
\hline & ps & 0,891 & 0,221 & 0,618 & 0,363 & 0,928 & 0,927 & 0,089 & 0,297 & 0,496 & 0,388 & 0,273 & 0,293 \\
\hline
\end{tabular}

Примечание: Статистическая достоверность между показателями:

род - в той же группе с показателями обычного дня (парное - по критерию Wilcoxon); рдэ - в той же группе с показателями перед экзаменами (парное - по критерию Wilcoxon). pf - с показателями группы флегматиков (ранговое, по критерию Mann-Whitney); px - с показателями группы холериков (ранговое, по критерию Mann-Whitney); ps - с показателями группы сангвиников (ранговое, по критерию Mann-Whitney); p17 - со статистическими данными 17-летних.

Полученные результаты показывают, чтоэмоциональная напряженность как в период ДЭ, так и ПЭ не оказывает ни усиливающего, ни ослабляющего действия на амплитуду волн в правой части теменной доли головного мозга. Поэтому в течении каждого из двух экзаменационных процессов амплитуда волн не изменяется.Вероятнее всего, адаптационный процесс у студентов в этом возрасте уже полностью сформирован. С другой стороны, благодаря тому, что данные студенты в течении учеб- 
ного процесса регулярно участвуют в экзаменационных процессах, у них ослабевают элементы ситуативного волнения, и в отличие от 17-летних действие эмоционального стресса на центральную нервную систему находится на более низком уровне. В конечном итоге, в каждой из двух теменных долей головного мозга сила амплитуды ЭЭГ волн во всех группах практически не изменяется.

У 21-летних юношей меланхолического типа в правой и левой частях теменной доли головного мозга амплитуда дельта-, тета-, альфа- и бета-волн в сравнении с соответствующими показателями других типов в течении всего исследованияпродемонстрировали неоднозначные различия.Такие различия хорошо заметны в амплитуде бета волн в период ПЭ $(\mathrm{P}<0,05)$. При сравнении студентов меланхолического типа с флегматическим выяснилось, что в периодыДЭ и ПЭ в бета-волнах левой и правой теменных долей имела место определенная разница. Аналогичная закономерность прослеживалась в бета-волнах в теменной доле головного мозга при сравнении меланхолического типа с холерикамии сангвиникамив период ПЭ. Разница наблюдалась также и в альфа-волнах в периоде ДЭ. В левой теменной доле у студентов меланхолическогои холерического типа разница чувствовалась в альфа- волнах в периоде ДЭ, вдельтаи альфа-волнах в периоде ПЭ. При сравнении меланхолического типа с сангвиниками в период ПЭ отмечалась разница в альфа-волнах левой теменной доли. В правой теменной доле в периодах ОД, ДЭ и ПЭ различий в волнах не наблюдалось.

\section{Заключение}

Результаты проведенного исследования показывают, что если в ритмах амплитуды волн ЭЭГ у 21-летних (Vкурс) меланхоликов в правой и левой частях теменной доли головного мозга разница не обнаруживается, то у 17-летних студентов I-го курса в амплитуде всех волн выявляются резкие перепады. В периоды высокого эмоционального стресса ДЭ и ПЭ в обеих частях теменной доли увеличивается ритм амплитуды дельта -волн ЭЭГ $[4,5,7,9,11]$.Во время покоя ритм альфа-волн ЭЭГ слабо изменяется. Эта разница более заметна в период ДЭ. У всех студентов младших курсов во время ожидания результатов экзамена тревожная ситуация еще более усиливается $[2,3,6,11]$.

Поэтому в теменной доле головного мозга у меланхоликов в период ДЭ и ПЭ в связи с волнением ритм волн ЭЭГ повышается. Однако, в амплитуде бета-волн наблюдается резкое ослабление. С другой стороны, в 
экзаменационной ситуации мотивы развития эмоционального напряжения у разных студентов повышаются в разной степени. Первопричина этого, в том, что студенты I-го курса впервые участвуют в экзаменах, в связи с чем у них отсутствует уверенность в достижении необходимых результатов. Фактически, экзамен будучи состоянием психоэмоционального напряжения, по-разному оказывает воздействие на функционирование организма. Возникновение эмоционального напряжения во время экзамена характеризует как субъективное, так и объективное состояния студента. Поэтому индивидуальное состояние тревожности у первокурсников в период ДЭ варьирует в широких пределах [11]. У студентов V-го курса в экзаменационной ситуации повышение активности нейрофизиологического коррелятора эмоционального напряжения в разных долях головного мозга,в отличие от тех же показателей у студентов 1-го курса, между группами практически не изменяется $[12,13]$. Перед экзаменами (ДЭ) у студентов с высокими по тесту Льюшера показателями стресса и с повышенной тревожной реактивностью по тесту Спилберга те или иные ЭЭГ ритмы в теменной доле головного мозга достоверно высокие $[4,5,7,8,14]$. Однако даже у таких студентов V-го курса степень адаптации к эмоциональному напряжению, возникшему во время экзаменационной ситуации, бывает высокой. В отличие от этого, у 21-летних студентов -меланхоликов амплитуда бета-волн ЭЭГ в правой и левой теменной доле головного мозга резко возрастает.У данных студентов происходит определенное ослабление формирования адаптации к эмоциональному напряжению в период экзаменов [11, 18].

Таким образом,важность проблемы, которую мы исследуем, обусловлена обострением противоречий между биопсихосоциальной сущностью человека и воздействием неудовлетворительных психосоциальных, экологических факторов, которые в последние годы все чаще приводят к нарушениям адаптации и развитию стресса. В связи с этим в исследованиях можно попытаться объяснить роль темперамента в стрессовых воздействиях путем обследования студентов разных курсов с использованием методов психофизиологического тестирования и электрофизиологических методов в условиях экзаменационного стресса, который является моделью эмоционального напряжения.Поскольку изучение системных механизмов интеграции физиологических функций в экзаменационных процессах у студентов является одной из важнейших задач современной возрастной физиологии, биомедицины в целом, реализация этих вопросов в течение образовательного процесса тесно связана с необходимостью разработки 
теоретических критериев абсолютных значений физиологической нормы иотклонений, отражающих тесную системную взаимосвязь функций.

\section{Выводы}

У 17-летних студентов с меланхолическим типом нервной системы в периоды ДЭ и ПЭ в обеих теменных долях головного мозга от воздействия тревожной ситуации эмоционального напряжения амплитуда дельта-, тетаи альфа-волн ЭЭГ в I семестре изменялась по нарастающей. Но у этих же студентов бета- волны изменялись двухфазно.

1. В процессе экзаменов от воздействия эмоционального напряжения у обучающихся на I и V курсах молодых людей меланхолического типа в левой и правой теменных долях головного мозга в ритме амплитуды волн ЭЭГ резких отклонений не происходит.

2. В экзаменационной ситуации у студентов V-го курса в отличие от студентов I-го курса нейрофизиологический коррелят эмоционального напряжения в зависимости от изменений активности в разных участках долей головного мозга между разными группами не различается.

3. Установлено, что в зависимости от темперамента нервной системы у студентов I-го и V-го курса в показателях электрической активности мозга есть существенная разница.

4. В зависимости от силы, подвижности и стабильности нервной системы, изменение ритмов амплитуды волн ЭЭГ впериод ДЭ и после бывает различным. В амплитуде большинства волн в период ПЭ наблюдается частичное снижение.

\section{Список литературы}

1. Айзенк Г.Ю. Количество измерений личности: 16.5 или 3 критерии таксономической парадигмы // Иностранная психология, 1993, Т. 1, № 2. С. 9-24.

2. Александров А.Г., Лукьяненок П.И. Изменение уровней тревожности студентов в условиях учебной деятельности // Научное образование. Медицинские науки, 2016, №6. С. 5-14.

3. Бартош О. П., Бартош Т. П. Социально-психологический тренинг и метод биологической обратной связи как профилактика тревожности у младших школьников // Вестн. психотерапии. 2016, 58 (63). С. 35-45.

4. Березнева Е.Ю., Крысова Т.И. Взаимосвязь мотивации учебной деятельности у подростков с видами темперамента, ситуативной тревожностью и межличностыми отношениями в семье // Международный журнал прикладных и фундаментальных исследований, 2015, №10-2. С. 357-360. 
5. Глазачев О.С., Классина С.Я., Бобылева О.В. Полисенсорная ритмическая афферентация в оптимизации психофизиологического статуса человека // XXI съезд Физиологического общества им. И.П.Павлова. Тезисы докладов. М.:, Калуга: «БЭСТ-Принт», 2010. С.144-145.

6. Двоеносов В.Г. Физиологическая характеристика адаптивных индивидуально-типологический реакций организма при действии экстремальных факторов. Дис....док.биол.наук, М., 2009. 354 с.

7. Искандерова Г.Т. Возрастная динамика психофизиологических показателей у юношей // Гигиена и санитария, 2006, №4. С.61-64 .

8. Курман О. А., Трубникова Л. В., Абдукаримов А. М. Влияние стрессового состояния на физиологические особенности учащихся во время пробного экзамена // Молодой ученый, 2018, №32. C. 22-24. URL: https://moluch.ru/ archve/218/52270/

9. Лила Н.Л. Особенности адаптивных воздействий студентов с разными индивидудально-типологическими характеристиками психической деятельности и вегетативной регуляции организма. Дис....канд.мед наук. Луганск, 2015. 147 с.

10. Сергеев Н. А. Влияние экзаменационного стресса на физиологические особенности организма учащихся девятого класса во время пробного экзамена по математике // Сборник исследовательских работ участников XXIII Всероссийского Конкурса юношеских исследовательских работ им. В. И. Вернадского. М., 2016. С. 46.

11. Тарасова С. Ю., Осницкий А. К. Психофизиологические и поведенческие показатели школьной тревожности // Психол. Наука и образование. 2015, Т. 20, №1. C.59-68.

12. Трошин В.Д. Стресс и стрессогенные расстройства. М.: МИА, 2007. 779 с.

13. Тумялис А.В., Коренек В.В., Махнев В.П. и др. Индивидуальная частота альфа-активности и переживание положительных и отрицательных эмоций // XXI Съезд Физиологического общества им. И.П.Павлова. Тезисы докладов. М., Калуга: БЭСТ-Принт, 2010. С. 622-623.

14. Умрюхин Е. А., Джебраилова Т.Д., Коробейникова И.И. и др. Связь результативности целенаправленной деятельности с параметрами ЭЭГ студентов в ситуации экзаменационного стресса // Психологический журнал. 2003, T. 24, №3. C. 88-93.

15. Bazanova O.M., Aftanas L.I. Individual EEG Alpha Activity Analysis for Enhancement Neurofeedback Efficiency: Two Case Studies // Journal of Neurotherapy. 2010. T. 14. № 3. C. 244-253.

16. Bratsas C., Papadelis C., Konstantinidis E., Pappas C. Toward Emotion Aware Computing: An Integrated Approach Using Multichannel Neurophysiological 
Recordings and Affective Visual Stimuli // IEEE transactions on information technology in biomedicine. 2010. Vol. 14, № 3. P.589-597.

17. Fingelkurts A.A., Fingelkurts A.A., Rytsala H. et al. Impaired functional connectivity at EEG alpha and theta frequency bands in major depression // Hum. Brain Mapp. 2007. Vol. 28, № 3. P.247-261.

18. Hayden E.P., Shankman S.A., Olino Th.M. et al. Cognitive and temperamental vulnerability to depression: Longitudinal associations with regional cortical activity // Cognition \&Emotion. 2008. Vol. 22, № 7. P.1415-1428.

19. Schwarzer Ch., Buchwald P. Examination stress: measurement and coping // An xiety, Stress \& Coping: An Intern. Jour., 1477-2205, vol. 16, Issue 3, 2003, p. 247-249.

20. Spielberger C.D., Ritterband L.M., Sydeman S.J., Reheiser E.C. Assessment of emotional states and personality traits: measuring psychological vital signs // Clinical Personality Assessment: Practical Approaches. New York: Oxford University Press, 1995, p. 42-58.

\section{References}

1. Eysenck G.Yu. Inostrannaya psikhologiya [Foreign Psychology], 1993, V. 1, No. 2. P. 9-24.

2. Alexandrov A.G., Lukyanenok P.I. Nauchnoe obrazovanie. Meditsinskie nauki [Scientific education. Medical Sciences], 2016, No. 6. P. 5-14.

3. Bartosh O.P., Bartosh T.P. Vestnik Psikhoterapii [Bulletin of psychotherapy]. 2016, 58 (63). P. 35-45.

4. Berezneva E.Yu., Krysova T.I. Mezhdunarodnyy zhurnal prikladnykh i fundamental'nykh issledovaniy [International Journal of Applied and Fundamental Research], 2015, No. 10-2. P. 357-360.

5. Glazachev O.S., Klassina S.Ya., Bobyleva O.V. Polisensornaya ritmi-cheskaya af $\neg$ ferentatsiya v optimizatsii psikhofiziologicheskogo statusa cheloveka [Polysensory rhythmic afferentation in the optimization of the psychophysiological status of a person]. XXI sezd Fiziologicheskogo obshchestva im. I.P.Pavlova [XXI Congress of the Physiological Society of the I.P. Pavlova]. Abstracts of reports. M.: Kaluga: "BEST-Print", 2010. P.144-145.

6. Dvoenosov V.G. Fiziologicheskaya kharakteristika adaptivnykh individual'no-tipologicheskiy reaktsiy organizma pri deystvii ekstremal'nykh faktorov [Physiological characteristics of adaptive individual-typological reactions of the body under the action of extreme factors]. Dis .... doc. Biol. Sciences, M., 2009.354 p.

7. Iskanderova G.T. Gigiena i sanitariya [Hygiene and Sanitation], 2006, №4. P.61-64. 
8. Kurman O. A., Trubnikova L. V., Abdukarimov A. M. Molodoy ucheny [Young Scientist], 2018, No. 32. P. 22-24. URL: https://moluch.ru/archve/218/52270/

9. Leela N.L. Osobennosti adaptivnykh vozdeystviy studentov s raznymi individudal'no-tipologicheskimi kharakteristikami psikhicheskoy deyatel'nosti $i$ vegetativnoy regulyatsii organizma [Features of adaptive influences of students with different individual-typological characteristics of mental activity and autonomic regulation of the body]. Dis .... Candidate of Medical Sciences. Lugansk, $2015.147 \mathrm{p}$.

10. Sergeev N.A. Vliyanie ekzamenatsionnogo stressa na fiziologicheskie osobennosti organizma uchashchikhsya devyatogo klassa vo vremya probnogo ekzamena po matematike [Influence of examination stress on the physiological characteristics of the organism of ninth grade students during a test exam in mathematics]. Sbornik issledovatel'skikh rabot uchastnikov XXIII Vserossiyskogo Konkursa yunosheskikh issledovatel'skikh rabot im. V. I. Vernadskogo [Collection of research papers of participants of the XXIII All-Russian Competition for youth research works named after V. V.I. Vernadsky]. M., 2016. P. 46.

11. Tarasova S. Yu., Osnitskiy A.K. Psikhol. Nauka i obrazovanie [Psychol. Science and education]. 2015, V. 20, No. 1. P.59-68.

12. Troshin V.D. Stress i stressogennye rasstroystva [Stress and stress disorder]. M.: MIA, $2007.779 \mathrm{p}$.

13. Tumyalis A.V., Korenek V.V., Makhnev V.P. et al. Individual'naya chastota al'fa-aktivnosti i perezhivanie polozhitel'nykh i otritsatel'nykh emotsiy [Individual frequency of alpha activity and the experience of positive and negative emotions]. XXI Sezd Fiziologicheskogo obshchestva im. I.P.Pavlova [XXI Congress of the Physiological Society of I.P. Pavlova]. Abstracts of reports. M., Kaluga: BEST-Print, 2010. P. 622-623.

14. Umryukhin E. A., Dzhebrailova T.D., Korobeynikova I.I. et al. Psikhologicheskiy zhurnal [Psychological journal]. 2003, V. 24, № 3. P. 88-93.

15. Bazanova O.M., Aftanas L.I. Individual EEG Alpha Activity Analysis for Enhancement Neurofeedback Efficiency: Two Case Studies. Journal of Neurotherapy. 2010. V. 14. № 3. P. 244-253.

16. Bratsas C., Papadelis C., Konstantinidis E., Pappas C. Toward Emotion Aware Computing: An Integrated Approach Using Multichannel Neurophysiological Recordings and Affective Visual Stimuli. IEEE transactions on information technology in biomedicine. 2010. Vol. 14, № 3. P. 589-597.

17. Fingelkurts A.A., Fingelkurts A.A., Rytsala H. et al. Impaired functional connectivity at EEG alpha and theta frequency bands in major depression. Hum. Brain Mapp. 2007. Vol. 28, № 3. P. 247-261. 
18. Hayden E.P., Shankman S.A., Olino Th.M. et al. Cognitive and temperamental vulnerability to depression: Longitudinal associations with regional cortical activity. Cognition \&Emotion. 2008. Vol. 22, № 7. P. 1415-1428.

19. Schwarzer Ch., Buchwald P. Examination stress: measurement and coping. An xiety, Stress \& Coping: An Intern. Jour., 1477-2205, vol. 16, Issue 3, 2003, P. 247-249.

20. Spielberger C.D., Ritterband L.M., Sydeman S.J., Reheiser E.C. Assessment of emotional states and personality traits: measuring psychological vital signs. Clinical Personality Assessment: Practical Approaches. New York: Oxford University Press, 1995, P. 42-58.

\section{ДАННЫЕ ОБ АВТОРЕ}

Рустамова Тюкязбан Вагиф кызы, доктор наук, доцент кафедры Анатомии, физиологии и зоологии

Гянджинский государственный университет

пр. Гейдара Алиева 425, г. Гянджа, AZ2001, Азербайджан

rustamovatukezban72@mail.ru

\section{DATA ABOUT THE AUTHOR}

Rustamova Tukazban, Dr. Sc., Associate Professor of the Department of Anatomy, Physiology and Zoology

Ganja State University

425, Heydar Aliyev ave., Ganja, AZ2001, Azerbaijan

rustamovatukezban72@mail.ru 